УДК 512.544

\title{
On Some Properties of Adjoint Groups of Associative Nil Algebras
}

\author{
Anatoliy I. Sozutov \\ Inna O. Alexandrova* \\ Institute of Mathematics and Computer Science \\ Siberian Federal University \\ Svobodny, 79, Krasnoyarsk, 660041
}

Russia

Received 13.09.2016, received in revised form 15.03.2017, accepted 23.08.2017

Local stepped configuration of adjoint groups of associated nil algebras is proved. Problems 8.67, 9.76, 13.53 from Kourovka Notebook are partially solved. A number of new issues are formulated.

Keywords: associative algebra, adjoint group, locally step group.

DOI: 10.17516/1997-1397-2017-10-4-503-508.

According to A. I. Maltsev [1], group G is termed the generalized nilpotent group or $N$-group if the intersection of all the members of its lower central series is equal to one. Associative algebra A is termed the generalized nilpotent or $N$-algebra if the intersection of all its finite degrees is equal to zero. It is easy to verify that adjoint groups of $N$-algebras and all of their subgroups are $N$-groups [1]. Finitely generated infinite nil algebras proposed by E. S. Golod [2] and their adjoint groups are generalized nilpotent algebras. Further development of the method presented in [2] has resuted in the construction of finitely generated not finitely approximated associative nil algebras and groups [3] (see [4, pp. 56-61]) and even simple nil algebras generated by a countable number [5]. It is shown in this paper that adjoint groups of associate nil algebras satisfies weaker condition than residual finiteness introduced by S. N. Chernikov [6].

Theorem 1. Adjoint groups of associate nil algebras are locally graded.

Theorem 2. Let us assume that $A$ and $B$ are subsets of infinite locally graded p-group $G=$ $\langle A, B\rangle$. Then subgroup $\left\langle A^{x}, B^{y}\right\rangle$ is infinite for any $x, y \in G$.

Solution to problem 13.53 from [7] for locally graded $p$-groups:

Corollary 1. Let $G=\langle a, b\rangle$ be infinite locally graded p-group. Then subgroup $\left\langle a, b^{g}\right\rangle$ is infinite for any element $g \in G$.

Theorem 3. A primary locally graded $F^{*}$-group is biprimitive finite group.

Partial solution to problems 8.67 and 9.76 from Kourovka Notebook follows from Theorem 3 and the main result of [8].

Corollary 2. Each element of infinite non-Chernikov locally graded primary $F^{*}$-group $G$ is contained in some infinite abelian subgroup.

* aio40@mail.ru

(c) Siberian Federal University. All rights reserved 


\section{Definitions and auxiliary propositions}

The group is termed the locally graded if every infinite finitely generated subgroup contains the proper subgroup of finite index [6].

A group $G$ is termed the weakly biprimitive finite if any of its two elements of the same prime order generate a finite subgroup. A group $G$ is termed the biprimitive finite if this property is inherited by all its sections by finite subgroups [8]. For example, $d$-generated Golod groups $(d \geqslant 3)$ with $(d-1)$-generated finite subgroups are biprimitive finite groups [2].

Group $G$ is termed the $F$-group if for any of its finite subgroup $K$ and any elements $a, b$ of the same prime order from $T=N_{G}(K) / K$ there exists such element $c \in T$ that $\left\langle a, b^{c}\right\rangle$ is finite. If this property is inherited by subgroups of group $G$ then group $G$ is termed the $F *$-group (see in $[7,7.42])$. The reason to introduce such a weak condition of finiteness is infinite Olshansky group [9]. All proper subgroups of Olshansky group have the same simple order $p$, and they are conjugated.

Let $A$ be associative algebra over a field $\Phi$. For any subsets $X, Y \subset A$ a subspace spanned by the elements $x \in X, y \in Y$ is denoted as $X Y$. Subspaces $A^{2}=A \cdot A$ and $A^{n}=A^{n-1} \cdot A=A \cdot A^{n-1}$ (degrees of algebra $A$ ) are subalgebras and ideals of algebra $A$. We need two propositions that are proved in [10].

Proposition 1. Let us assume that for some elements $g_{1}, \ldots, g_{k}$ of the associative nil algebra A equalities

$$
g_{i}=\sum_{j=1}^{k} g_{j} v_{i j}, \text { where } i=1, \ldots, k \text { and } v_{i j} \in A
$$

are satisfied. Then $g_{1}=g_{2}=\ldots=g_{k}=0$.

Proposition 2. If associative nil algebra $A$ is finitely generated then $A \neq A^{2}$ and subalgebra $A^{2}$ is finitely generated.

Invertible elements of algebra $A$ with respect to the operation of adjoint multiplication $a * b=$ $a+b+a b$ form an adjoint group with zero element as the unit of the group [1]. When $A$ is a nil algebra, i.e $a^{n}=0$ for any $a \in A$ and natural $n=n(a)$, every element in $A$ is invertible with respect to the operation $*$, and when $\operatorname{Char} \Phi=p \neq 0$ adjoint group $A(*)$ is an $p$-group.

Lemma 1. In the adjoint group $G=A(*)$ of infinite-dimensional associative nil algebra $A$ each element is contained in the infinite-dimensional abelian subgroup.

Proof. Annihilator Ann $g$ of each element $g$ in nil algebra $A$ is infinite-dimensional because the chain $A \geqslant g A \geqslant g^{2} A \geqslant l$ dot of subalgebras $g^{k} A$ is finite, and it ends with zero subalgebra. Since $g * h=g+h+g h=g+h=h * g$ for $h \in \operatorname{Ann} g$ the subgroup $C_{G}(g)$ is infinite for every $g \in G$. This proves the lemma.

Solution of problems 8.67 and 9.76 for the adjoint group $A(*)$ from the Kourovka notebook follows from Lemma 1 [11]. A subgroup $P=\left\langle x_{1}, \ldots, x_{d}\right\rangle$ of group $A(*)$ generated by the same elements that algebra $A$ is termed the main subgroup.

Lemma 2. If in infinite p-group $Q$ the centralizer of each not unit element is finite then all maximal locally finite subgroups in $Q$ are finite and they form splitting of the group $Q$. In particular, any two elements selected from the various components of this splitting generate infinite subgroup in $Q$.

Proof. Since each infinitely local subgroup has an infinite abelian subgroup then all maximal locally finite subgroups in $P$ are finite and, therefore, they are nilpotent. With the help of the process of normalization it is easy to see that the intersection of any pair of maximal finite 
subgroups in $Q$ is the unit. Therefore, the splitting of the group $Q$ is all maximal finite subgroup $Q$, and any pair of elements selected from the different components of this splitting generates an infinite subgroup. The proof is complete.

Lemma 3. Let $P$ be d-generated p-group and, $D$ is intersection of all its maximal subgroups of finite index. Let us assume that $D \neq P$. Then

1) $D$ is normal in $P$ and $P / D$ is finite elementary abelian p-group of the rank $r \leqslant d$;

2) if subgroup $M$ is maximal in $P$ and $[P: M]=\infty$ then $P=M D$;

3) if $H$ is proper subgroup in $P$ and $P=H D$ then group $P$ is infinite and $P=H K$ for every subgroup $K$ of finite index from $P$.

Proof. 1. According to conditions of the lemma, the set of maximal subgroups in $P$ group with finite index in $P$ is not empty. Let $M$ be one of such subgroups. According to the Poincare theorem [12], index of normal in $P$ subgroup $N=\cap_{x \in P} M^{x}$ is finite. Hence, $P / N$ is a finite $p$ group and subgroup $M / N$ is maximal subgroup in the factor group $P / N$. According to Theorem $[13,4.3 .2][P / N: M / N]=p$ and $M / N$ is normal subgroup in $P / N$. According to Theorem $[13,2.3 .4] M$ is normal subgroup in $P$ and $[P: M]=p$. In particular, $M=N$ and factor-group $P / M$ is a cyclic group of order $p$. Consequently, elements in $P$ to the power $p$ and all commutators are in the subgroup $M$. Hence, subgroup $D$ is normal subgroup in $P$, and it contains any element from $P$ to the power $p$ and the commutator $[x, y]$ of any two elements $x, y$ from $P$. Therefore, the factor group $P / D$ is elementary abelian group. Since $P$ is finitely generated group then $P / D$ is finitely generated group and it is a finite group of order $p^{r} \leqslant p^{d}$. The statement is proved.

2. It is clear that $D \nless M$. Because $D \triangleleft P$ (see above) then the set $M D$ is a group. By the maximality of $M$ we have $M D=P$. The statement is proved.

3. Let $P=H D$, where $H$ is a proper subgroup of $P$. According to Burnside theorem on basis (eg, [13, Theorem 12.2.1]), this situation is impossible in finite groups then group $P$ is infinite. Let $K$ be arbitrary subgroup of finite index in $P$ and $L=K \cap D$. According to the Poincare theorem [12] the index of subgroup $L$ in $P$ is finite, and the index of normal subgroup in $P N=\cap_{x \in P} L^{x}$ is also finite. It is obviously that $D / N$ is a Frattini subgroup of the factor group $P / N$ and $P / N=D / N \cdot H N / N$ by the condition $P=H D$. Theorem 12.2.1 [13] gives $P / N=H N / N$. Hence we have $H N=P$ and $H K=P$. Lemma is proved.

\section{The Proof of Theorems}

Proof of Theorem 1. Let $R(*)$ be an adjoint group of arbitrary associative nil algebra $R$ over the field $\Phi, k$ is a natural number and $H$ is an arbitrary infinite subgroup of $R(*)$ generated by elements $g_{1}, \ldots, g_{k}$. We need to prove that $H$ contains a proper subgroup of a finite index. It is clear that the linear hull $A$ of set $H$ is $k$-generated subalgebra of algebra $R$, and $H$ is the main subgroup of the adjoint group $A(*)$. Accordind to Proposition $2, A^{2}$ is a proper ideal of algebra $A$, and consequently the subgroup $A^{2}(*)$ is normal in $A(*)$ [1]. Since $a * b+A^{2}=b * a+A^{2}$ for any $a, b \in A$ then factor group $A(*) / A^{2}(*)$ is an elementary abelian $p$-group in the case of Char $\Phi=p \neq 0$, and it is abelian group without torsion in the case of Char $\Phi=0$. It follows from proposition 1 that at least one element $g_{1}, \ldots, g_{k}$ does not belong to the subalgebra $A^{2}$, and $D=H \cap A^{2}(*)$ is a proper normal subgroup in $H$. Since the factor group $A(*) / A^{2}(*)$ is abelian group, the factor group $H / D$ is a finitely generated abelian group. If Char $\Phi=p \neq 0$ the factor group $H / D$ is an elementary abelian $p$-group of order $\leqslant p^{k}$ and $D$ is required proper subgroup of finite index of $H$. If Char $\Phi=0$ and taking into account the fundamental theorem on finitely generated abelian groups [14], the factor group $H / D$ is the direct product of a finite number of cyclic groups. It is easy to verify that in this case there is a proper subgroup $M / D$ of finite index 
in $H / D$, and its complete inverse image $M$ is a proper subgroup of finite index in group $H$. The theorem is proved.

Proof of Theorem 2. In the case where at least one of the sets $A, B$ is infinite there is nothing to prove. Let us assume that $A, B$ are finite non-empty subset, $x, y$ are arbitrary elements of $G$ and $H=\left\langle A^{x}, B^{y}\right\rangle$. Since $G$ is locally graded and finitely generated then the set of proper subgroups of finite index of $G$ is non-empty. Therefore, the intersection $D$ of all its maximal subgroups of finite index differs from $G$. Because subgroup $D$ is normal in $G$ (see Lemma 3) it follows from conditions of the theorem that $G / D=\langle A D / D, B D / D\rangle$. According to Lemma 3, the factor group $G / D$ is abelian group, and for all elements $x, y \in G$ equalities $A D / D=A^{x} D / D$, $B D / D=B^{y} D / D$ hold. Hence we have $H D / D=G / D$ and $H D=G$. According to Lemma 3, $G=H K$ for every subgroup $K$ of finite index in $G$. According to Lemma [15, 1.15], $G$ has infinite strictly decreasing series

$$
G=G_{0}>G_{1}>\cdots>G_{i}>\cdots
$$

of finitely generated characteristic subgroups of finite index. Taking into account the proof given above, $G=H G_{i}$ for all parts $G_{i}, i=1,2, \ldots$, of series (1). Because indexes $\left[G: G_{i}\right]$ of members $G_{i}$ of series (1) are not bounded in total and $\left[G: G_{i}\right]=\left[H: H \cap G_{i}\right]$ then subgroup $H$ cannot be finite. The Theorem is proved.

Proof of Theorem 3. Let the $p$-group $G$ satisfies given conditions. If some pair of elements $a, b$ of order $p$ from $G$ generates a infinite subgroup $H$ then by Corollary 1 subgroup $\left\langle a, b^{c}\right\rangle$ is infinite for any element $c \in H$. This is in contradiction with the definition of $F^{*}$-group. Therefore, any pair of elements of order $p$ generates a finite subgroup in $G$, and the group $G$ is finite weakly biprimitive group. Let $K$ be any finite subgroup of $G$ and $T=N_{G}(K) / K$. Let us assume that there are elements $\bar{a}, \bar{b}$ of order $p$ in $T$ that generate infinite subgroup. Then subgroup $H=\langle a, b\rangle$ is infinite too (here $a, b \in G$ and $a K=\bar{a}, b K=\bar{b}$ ). By Corollary refs1 subgroup $\left\langle a, b^{c}\right\rangle$ is infinite for any $c \in H$. Then all subgroups $\left\langle\bar{a}, \bar{b}^{\bar{c}}\right\rangle$ are infinite, where $\bar{c} \in H K / K$. This is in contradiction with the definition of $F^{*}$-group. Hence, any pair of elements of order $p$ generates a finite subgroup in $T$ and group $G$ is biprimitive finite group. The theorem is proved.

\section{Some questions about the structure of the main group}

Let $F=\Phi\left(x_{1}, \ldots, x_{d}\right)$ be free associative algebra of polynomials over the field $\Phi$ with respect to noncommuting variables $x_{1}, \ldots, x_{d}$. Algebra $F$ is graded, because it decomposes into a direct sum $F=F_{0} \oplus F_{1} \oplus \ldots$ of subspaces $F_{n}$ with $F_{i} F_{j} \subseteq F_{i+j}$, where $F_{0} \simeq \Phi$ and $F_{n}$ is the subspace of dimension $d^{n}$ with the basis of monomials $x_{i_{1}} x_{i_{2}} \ldots x_{i_{n}}$. Let us consider the infinite-dimensional algebras $A=\left\langle\left\langle x_{1}, \ldots, x_{d}\right\rangle\right\rangle=\Phi\left(x_{1}, \ldots, x_{d} \mid f_{1}, f_{2}, \ldots\right)$. Generating elements $x_{1}, \ldots, x_{d}$ of algebra $A$ are on the left of the line. Defining relations

$$
f_{1}, f_{2}, \ldots-\text { polynomials of } F_{2} \oplus F_{3} \oplus \ldots---
$$

are on the right of the line. They generate the ideal $J, A \simeq F^{\prime} / J$, where $F^{\prime}=F_{1} \oplus F_{2} \oplus \ldots$ If $x_{1}, \ldots, x_{d}$ are nilelements in $A$ then submonoid $P=\left\langle x_{1}, \ldots, x_{d}\right\rangle$ in the attached monoid $A(*)$ is a group. In the formula of the adjoint multiplication $a * b=a+b+a b$ nonidentical field elements are absent. Therefore, upon ignoring the potentially possible linear relations between elements of the group $P$ over the field $\Phi$ further, we assume that the field $\Phi$ is simple and it coincides with the residue ring $Z_{p}$. Algebra $\Phi \oplus A$ is a homomorphic image of the group algebra $\Phi[P]$, and therefore $P$ is an infinite group [2,14]. Question: When the set $P \backslash\{0\}$ is the basis of algebra $A$ ?

Let $A=\left\langle\left\langle x_{1}, \ldots, x_{d}\right\rangle\right\rangle$ be infinite nil algebra. Is the index of the subgroup $P$ in $G=A(*)$ finite or infinite? Is it true that every finitely generated subgroup of group $G$ has infinite index 
in $G$ ? There is the question in connection with Lemma 3: Can a maximal subgroup $M$ of group $P$ have infinite index in $P$ ?

Necessary and sufficient conditions for the embeddability of a primary $N$-group attached to the group of some $N$-algebra [1]: In order that $N$-group $H$ be embedded isomorphic to a affiliate group of some $N$-algebra over a field of characteristic $p>0$, it is necessary and sufficient that $H$ does not contain elements of infinite p-height. According to Theorem 1, group $G=A(*)$ and its main subgroup $P$ are locally graded, and the class of locally graded groups is much wider than the class of residually finite groups [15]. Upon considering Maltsev theorem and examples of not generalized nilpotent groups and nil algebras [3-5], the question arises: May the group $P$ include a quasi-cyclic subgroup?

Let us reformulate problems 8.67 and 9.76 from [7]. Partial solutions to these problems are given in this paper. Is the centralizer of some non-trivial element in the group $P$ infinite? Can the centralizer of some element in the group $P$ be infinite? It follows from Lemmas 1, 2, Theorem 3 and Proposition 2 of the paper that it is sufficient to solve those questions for groups (and algebras) with two generators. Groups with two generators are worthy of consideration and for other reasons too (for example, problems 11.56, 12.43 and etc from [7]). Perhaps one should start with the following problem: find explicit formulas for the coefficients of Hilbert series and their asymptotic behavior for associative algebras generated by two nil-elements.

As noted in [1], every ideal $I$ of algebra $A$ is a normal subgroup of group $G=A(*)$. In particular, subgroup $I \cap P$ is normal in $P$. Reverse matching is not obvious even in the case of locally nilpotent algebras [16]. It follows from Theorem 1 that $P$ has the strictly decreasing lower central series

$$
P=P_{0}>P_{1}>\cdots>P_{i}>\ldots,
$$

where $P_{i}=\gamma_{i}(P)=\left[\gamma_{i-1}(P), P\right]$. Let $L_{i}$ be the linear span $P_{i}$ in $A$. The authors do not know answers to the following questions. Is subalgebra $L_{1}$ ideal in $A$ ? Does $L_{1}$ coincide with $A^{2}$ ? Are subalgebras $L_{i}$ ideals in $A$ ? For what normal subgroups of group $P$ their linear spans are ideals of algebra $A$ ?

On the basis of Golod construction [2] the famous problem of the existence of a simple associative nilring was solved [5]. Developed in [5] simple nil algebra $R$ is countable, and it is not finite by Proposition 2. Because $R$ is not locally nilpotent then its adjoint group $R(*)$ is not locally finite. Is the group $R(*)$ simple? The following issues seem promising. Are there infinite simple binary nilpotent associative algebras? Are their associated groups simple? In case of positive answers to these questions problems $6.48,6.61$ and 11.100 from [7] will be also solved.

Let $G=\left\langle g_{1}, \ldots, g_{d}\right\rangle$ be an arbitrary infinite $p$-group, and $\Phi[G]$ is its group algebra. For arbitrary $F$ mapping $1 \rightarrow 1, x_{1} \rightarrow g_{1}-1, \ldots, x_{d} \rightarrow g_{n}-1$ extends to homomorphism $\varphi$ of algebra $F$ on algebra $\Phi[G]$. Let $J$ be the kernel of $\varphi$. The image of the ideal $F^{\prime}=F_{1} \oplus F_{2} \oplus \ldots$, with codimension 1 in $F$ is subalgebra $A=\left\langle\left\langle g_{1}-1, \ldots, g_{d}-1\right\rangle\right\rangle$ from $\Phi[G]$ generated by nilelements $g_{1}-1, \ldots, g_{d}-1$. Under what conditions on the group $G$ the algebra $A$ is nilideal in $\Phi[G]$ ? This question is of particular interest for residually finite $p$-groups.

The work was supported by RFBR grant (project no. 15-01-04897-a).

\section{References}

[1] A.I.Maltsev, Generalized nilpotent algebras and their associated groups, Mat. sbornik, 25(1949), 347-366 (in Russian).

[2] E.S.Golod, On nil-algebras and finitely approximable p-groups", Izv. Akad. Nauk SSSR Ser. Mat., 28(1964),no. 2, 273-276 (in Russian). 
[3] A.Z.Ananyin, Nil-algebra with non-radical tensor square, Sib. mat. jurnal, 26(1985), no. 2, 192-194 (in Russian).

[4] V.I.Senashov, V.P.Shunkov, Groups with limbs, Novosibirsk, Pub. house of SB RAS, 2001.

[5] A.Smoktunowicz, Simple nil ring exits, Comm. Algebra, 30(2002), 27-59.

[6] S.N.Chernikov, About the product groups of finite rank, Algebra i logica, 20(1981), no. 3, 315-329 (in Russian).

[7] Kourovka notebook: Unsolved problems in group theory, American Mathematical Society, 1983.

[8] V.P.Shunkov, On one class of p-groups, Algebra and logic, 9(1970), no. 4, 484-496 (in Russian).

[9] A.Y.Olshansky, An infinite group with subgroups of prime order, Izv. Akad. Nauk SSSR Ser. Mat., 44(1980), no. 2, 309-321 (in Russian).

[10] V.A.Sereda, A.I.Sozutov, On associative nil-algebras and groups of Golod, In misc. Proceedings of the XXI Inter-University Scientific and Technical Conference (april 2003), Krasnoyarsk, KrasGASA, 2003, 21-44 (in Russian).

[11] Y.P.Sysak, Abstracts of the 17th All-Russian Algebraic Conference, part 1, Minsk, 1983 (in Russian).

[12] A.I.Sozutov, N.M.Suchkov, N.G.Suchkova, Infinite groups with involutions, Krasnoyarsk, Siberian Federal University, 2011 (in Russian).

[13] M.Hall, Group theory, New York, MacMillan, Co., 1959.

[14] M.I.Kargapolov, Y.I.Merzlyakov, Basics of group theory, Moscow, Nauka, 1982 (in Russian).

[15] N.S.Chernikov, Groups that are decomposable into a product of commuting subgroups, Kiev, Naukova Dumka, 1987 (in Russian).

[16] V.M.Liauchuk, Some locally nilpotent rings and their adjoined groups, Mat. Zametki, 42(1987), no. 5, 631-641 (in Russian).

\title{
О некоторых свойствах присоединенных групп ассоциативных нильалгебр
}

\author{
Анатолий И. Созутов \\ Инна О. Александрова \\ Институт математики и фундаментальной информатики \\ Сибирский федеральный университет \\ Свободный, 79, Красноярск, 660041 \\ Россия
}

Доказана локальная ступенчатость присоединенных групп ассочиативных нилъалгебр, частично решены вопросы 8.67, 9.76, 13.53 из Коуровской тетради и поставлен ряд новых вопросов.

Ключевые слова: ассоциативная алгебра, ступенчатая группа, локально ступенчатая группа. 